\title{
Lousy Suggestion of Second-Line Therapy Against Helicobacter pylori
}

\author{
Amin Talebi Bezmin Abadi ${ }^{1, *}$ \\ ${ }^{1}$ Department of Bacteriology, Faculty of Medical Sciences, Tarbiat Modares University, Tehran, Iran \\ "Corresponding author: Amin Talebi Bezmin Abadi, Assistant Professor, Department of Bacteriology, Faculty of Medical Sciences, Tarbiat Modares University, P.O. Box 14115-111, \\ Tehran, Iran. Tel/Fax: +98-2182884883, E-mail: amin.talebi@modares.ac.ir
}

Received 2016 July 17; Revised 2017 May 21; Accepted 2017 September 18.

Keywords: Treatment, Gastric Infection, Helicobacter pylori

\section{Dear Editor,}

We read the article by Shahabimehr et al., investigating 96 Iranian patients undergone gastric endoscopy at Imam Khomeini hospital in Tehran, from 2013 to 2014 (1). However, some newly found points may enrich the proposed conclusion by the authors:

1- As authors reported, in terms of geographic discrepancies in the levels of metronidazole resistance both for national and international levels, even routine tests cannot provide useful information to help physicians due to lack of efficacy reported in the combination of triple therapy. Therefore, the main rationale for investigating antibiotic resistance of Helicobacter pylori in most of regions is under debates (2).

2- Despite predictable findings in this survey, investigating only a single antibiotic suggested to treat $H$. pylori is not clinically recommendable (3). We fully admitted that monotherapy is not sufficient to treat gastric infection; thus, it is always prescribed with clarithromycin or amoxicillin. In other words, antibiotic susceptibility data are essential to understand the efficacy of $H$. pylori eradication regimens, but this recommendation cannot be generalized in the case of single antibiotic.

3-Authors" unpublished data showed that glmM carries certain mutations that can easily influence the current PCR results. Therefore, it can explain low positive rate of the clinical samples. Other genes such as 16s rRNA can be more reliable options to be testified.

4- The rate of antibiotic resistance was $81.8 \%$ (18/22) in the study. In Tehran, with a population of 13 million people, only 22 subjects cannot provide a proper sample size to create reliable statistically significant data. Of course, a larger population with better sampling is required to examine the main objectives related to the antibiotic resistance. Hence, using the wording "Iranian population" may be confusing to the readers according the current findings.
Last, but not least, regardless of the current suggestion by the authors about urgent call for second-line therapy, it seems that clinicians can still think about first-line, but with the administration of different useful antibiotics such as clarithromycin, levofloxacin, or amoxicillin.

\section{Footnote}

\section{Conflict of Interest: None.}

\section{References}

1. Shahabimehr M, Alebouyeh M, Farzi N, Mahboubi A, Taslimi R, Zali MR. Resistance rate and minimum inhibitory concentration of metronidazole among helicobacter pylori strains in Tehran, Iran. Arch Clin Infect Dis. 2016;11(2). doi: 10.5812/archcid.34478.

2. Talebi Bezmin Abadi A. Therapy of Helicobacter pylori: present medley and future prospective. Biomed Res Int. 2014;2014:124607. doi: 10.1155/2014/124607. [PubMed: 24800203].

3. Talebi Bezmin Abadi A. Helicobacter pylori treatment: New perspectives using current experience. J Glob Antimicrob Resist. 2017;8:123-30. doi: 10.1016/j.jgar.2016.11.008. [PubMed: 28131855]. 